\title{
Multiple systemic arteries to pulmonary artery malformations: a case description
}

\author{
Xinge Cheng ${ }^{1,2 \#}$, Xiaoyong Zhang ${ }^{2 \#}$, Fujia Gu ${ }^{3}$, Chong Tian ${ }^{2}$, Rongpin Wang ${ }^{2}$, Jiaxiang Chen ${ }^{4}$, Jian Liu ${ }^{1,2}$, \\ Xianchun Zeng ${ }^{2}$ \\ ${ }^{1}$ Department of Graduate School, Zunyi Medical University, Zunyi, China; ${ }^{2}$ Department of Radiology, Guizhou Provincial People’s Hospital, Key \\ Laboratory of Intelligent Medical Imaging Analysis and Accurate Diagnosis of Guizhou Province, International Exemplary Cooperation Base of \\ Precision Imaging for Diagnosis and Treatment, Guiyang, China; ${ }^{3}$ Department of Interventional Radiology, Guizhou Provincial People's Hospital, \\ Guiyang, China; ${ }^{4}$ Guizhou University School of Medicine, Guiyang, China
}

"These authors contributed equally to this work.

Correspondence to: Xianchun Zeng. Department of Radiology, Guizhou Provincial People's Hospital, 83 Zhongshan East Rd, Guiyang 550002, China. Email: zengxianchun04@foxmail.com.

Submitted Jan 27, 2021. Accepted for publication Apr 23, 2021.

doi: 10.21037/qims-21-109

View this article at: http://dx.doi.org/10.21037/qims-21-109

\section{Introduction}

Connections between the systemic circulation and the pulmonary arterial systems are extremely rare. Multiple systemic artery-to-pulmonary artery (SA-PA) malformations, which can be congenital or non-congenital, are extremely rare in people without congenital heart disease. The non-congenital fistulas can develop in patients with inflammatory or infectious disease of the lung and pleura, cancer, or after trauma (1-3). Congenital SA-PA malformations can be detected in patients without any of the risk factors mentioned above. An SA-PA malformation can occur in single or multiple forms and connect the pulmonary vascular bed with the left internal thoracic and the left gastric arteries, the pericardiacophrenic branch of the left musculophrenic artery, the left bronchial artery, or others.

In previous reports, most of the SA-PA malformations cases have displayed signs of pulmonary hypertension (4). Here we describe a case of non-congenital multiple SAPA malformations in a patient with a history of pulmonary tuberculosis 20 years prior and their 2-year follow-up after embolizing the SA-PA.

A new technique for three-dimensional (3D) visualization of cross-sectional image data called cinematic rendering (CR) was introduced recently $(5,6)$. The technique models the real-life physical propagation of light, creating complex lighting effects, such as shadows, subsurface scattering, refraction, absorption, depth of field, and ambient occlusion $(7,8)$. Therefore, CR can provide higher quality and photorealistic images compared with conventional volumerendering techniques. In this report, we revealed multiple SA-PA malformations through the use of CR.

\section{Case presentation}

A 41-year-old female presented to the Pneumology Department with chest tightness, shortness of breath, and dyspnea lasting over 20 days. Physical examination was normal, and no abnormality was found in pulmonary function, cardiac ultrasound, and radiography (Figure 1A). The patient had experienced pulmonary tuberculosis 20 years ago, which had been cured by anti-tuberculosis treatment, and the latest chest computed tomography (CT) had shown no tuberculosis lesions. There was no obvious inducing factor for chest tightness, shortness of breath, dyspnea, and dizziness before attending the hospital.

At admission, electrocardiography and echocardiography showed normal results. According to the patient's clinical symptoms, priority was given to the investigation of heart disease or vascular disease, so multi-slice spiral computed 

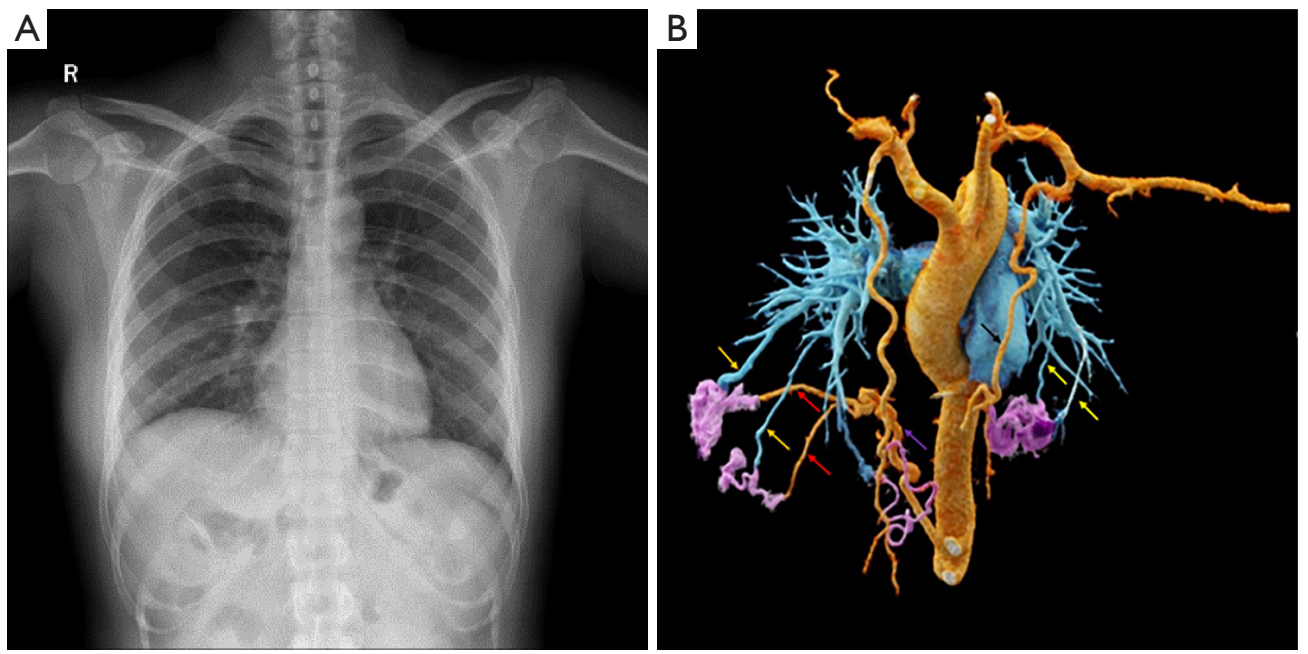

Figure 1 (A) Chest radiography with no abnormal findings. CR of the MSCTA, which offers a better view of the connection between the systemic artery and a pulmonary artery. (B) Different color arrows indicate the right musculophrenic artery (purple arrow), branch of the right musculophrenic artery (red arrow), right pulmonary artery (orange arrow), left internal thoracic artery (black arrow) and left pulmonary artery (yellow arrow). CR, cinematic rendering; MSCTA, multi-slice spiral computed tomography angiography
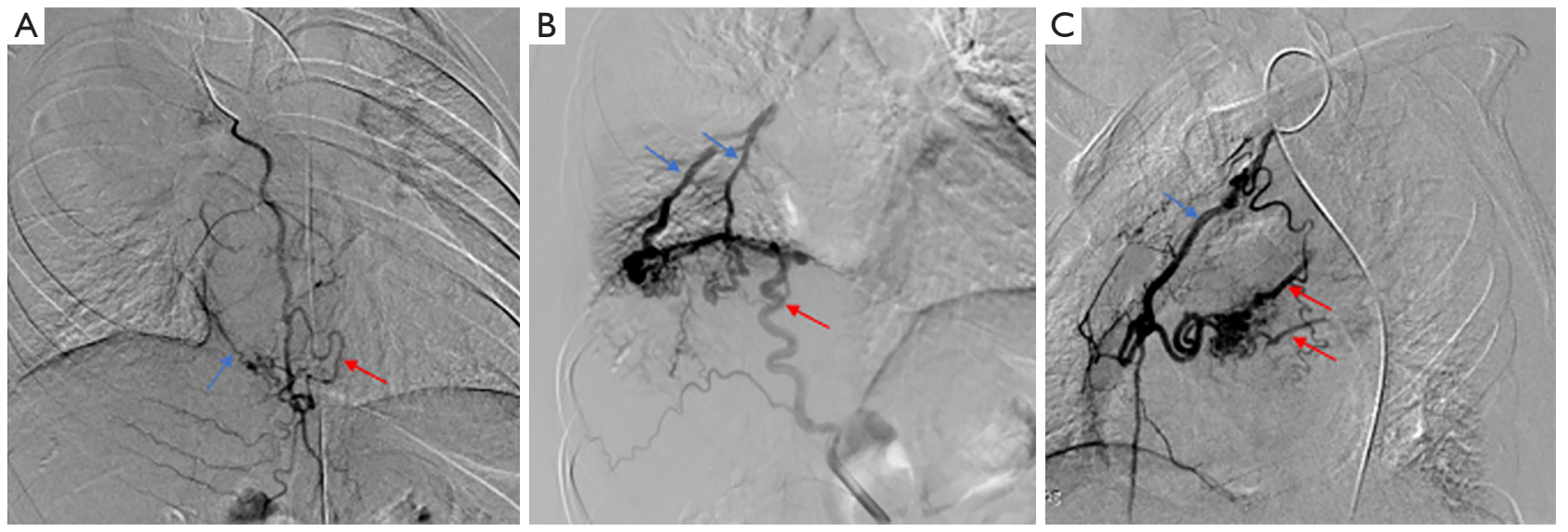

Figure 2 DSA results. (A) Selective angiography in an oblique view of the right internal thoracic artery (red arrow) and the right lower pulmonary artery branch (blue arrow) supply vascular malformation. (B) Selective right musculophrenic arteriogram in an oblique view demonstrates the malformation between the right musculophrenic artery (red arrow) and 2 branches of the right pulmonary artery (blue arrow). (C) Selective angiography in an oblique view of the left internal thoracic artery (blue arrow) and the left pulmonary artery (red arrow) supply vascular malformation. DSA, digital subtraction angiography

tomography angiography (MSCTA) examination was applied immediately, with CR to photo-realistically display the shape and number of vascular malformations. The CR revealed multiple connections between the bilateral internal thoracic arteries, right musculophrenic artery, and bilateral pulmonary artery branches (Figure $1 B$ ). This combination of features was highly suggestive of SA-PA malformation.
The patient underwent conventional angiography for further investigation. Digital subtraction angiography (DSA) showed multiple anomalous communicating vessels from several systemic arteries to the pulmonary circulation, including the bilateral internal thoracic arteries and the right musculophrenic artery (Figure 2), which were consistent with the manifestation of multiple SA-PA 


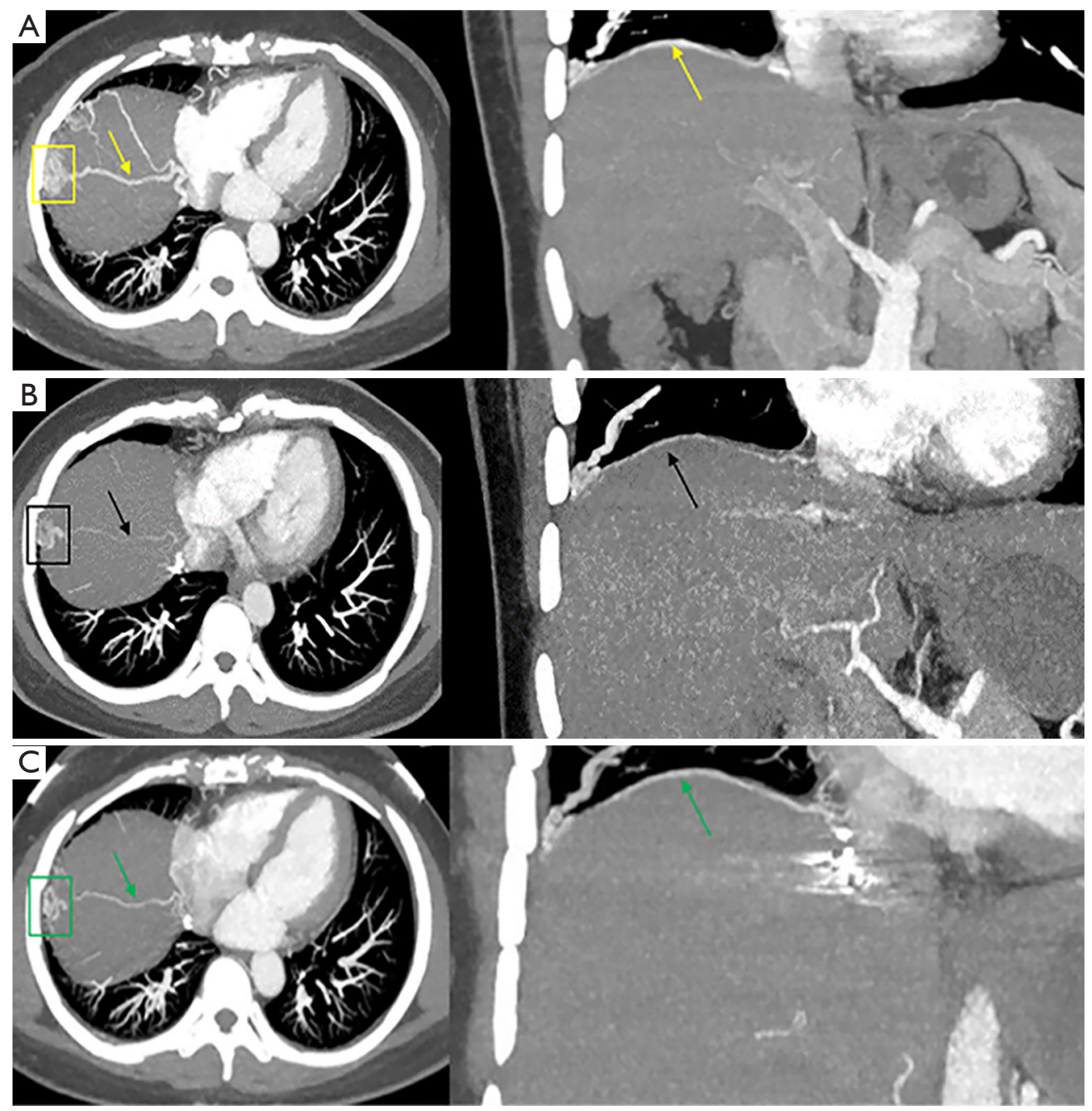

Figure 3 (A) Axial and coronal CT MIP reconstruction before embolization shows hypertrophy of the right musculophrenic artery (yellow arrow). (B) MIP reconstruction at 3 months after embolization shows atrophy of the right musculophrenic artery (black arrow). (C) The third MIP reconstruction at 2 years after embolization shows that the right musculophrenic artery had thickened compared to the image at the postoperative view. Yellow, black, and green frames indicate the vascular malformation in the right lower lobe. (Slice thickness: 15 mm, venous phase). CT, computed tomography; MIP, maximum intensity projection

malformations.

At the 2-year follow-up after embolization, the patient was asymptomatic and had no recurrence of clinical symptoms. However, MSCTA revealed slight hypertrophy of the right musculophrenic artery (Figure 3). Compared with the imaging results of the first review after embolization, this sign may have indicated the possibility of recurrence.

The study was conducted in accordance with the Declaration of Helsinki (as revised in 2013). Ethical approval was granted from the hospital's ethics committee, and written informed consent was provided by the patient for publication of this case report and any accompanying images. A copy of the written consent is available for review by the Editor-in-Chief of this journal.

\section{Discussion}

The SA-PA malformation represents a relatively rare vascular anomaly. The symptoms caused by SA-PA malformation are usually asymptomatic and vascular murmur can be discovered during routine physical 
examination (9). However, the physical examination of this patient was negative for such findings. It is possible that a deep location of vascular malformation or a smallsized vascular murmur made the detection of this vascular murmur difficult. Symptomatic patients usually present with symptoms of dyspnea, hemoptysis, or congestive heart failure (10). Here, the connection between systemic and pulmonary circulations may have been the cause of dyspnea in this patient. She was finally diagnosed with multiple SA-PA malformations by DSA. A challenge remains in distinguishing SA-PA malformation from several pulmonary vascular diseases.

Pulmonary arteriovenous malformations are direct connections between a pulmonary artery and vein branch through thin-walled aneurysmal sacs (11). In this case, selective angiography and MSCTA demonstrated no direct venous connection with any of the arteries that formed the vascular malformation.

Pulmonary sequestration is defined as a mass of abnormal pulmonary tissue that cannot communicate with the tracheobronchial tree through a normally located bronchus and is supplied by anomalous systemic arteries (12). In this case, this patient could not have been considered to have pulmonary sequestration because there was no sequestered mass of lung tissue.

Multiple SA-PA malformations are extremely rare in people with normal pulmonary circulation, and the detection of abnormal blood vessels is an important guide for making clinical treatment plans. Although selective angiography is the gold standard for diagnosis, the MSCTA scan is a useful tool for diagnosis and can provide complete anatomical details of the vascular malformation, thereby providing a reference for formulating DSA or surgical strategies.

Currently, the management of SA-PA malformation is challenging and controversial. The SA-PA malformation can be treated by surgery, embolization, or it can remain under observation. Historically, surgery was the primary treatment for SA-PA malformation, including resection of the anomalous vessels, lobectomy, and pneumonectomy. Recently, transcatheter embolization for the connecting vessels has been performed as a less invasive alternative therapy (5). This patient's vascular malformations were scattered and complicated, which might have caused complications such as pulmonary hypertension and massive hemoptysis, which can be life-threatening in the absence of timely treatment. However, radical removal of multiple reticular blood vessels may cause excessive bleeding during surgery (9). The risk of bleeding is directly related to the number of inflow arteries. After multidisciplinary team discussions, the risk of bleeding in surgical treatment was deemed high, and interventional embolization was also more difficult. Finally, the patient underwent interventional embolization in another hospital. However, this treatment is associated with recurrence, so repeated treatment might be required in the future. At the 2-year follow-up after embolization, this patient had no recurrence of clinical symptoms, while the MSCT imaging examination revealed slight hypertrophy of the right musculophrenic artery (Figure 3), signaling the possibility of recurrence. However, longer follow-up is needed to fully evaluate the efficacy of embolization.

In conclusion, DSA is the gold standard for diagnosing SA-PA malformation, but MSCTA can provide complete anatomical details of the vascular malformation, which may provide a reference for formulating treatment strategies. Additionally, CR can present CT volume data more photorealistically and naturally.

\section{Acknowledgments}

Funding: This study was supported by the Guizhou Science and Technology Project \{QKHZC[2020]4Y002\}; and the Guiyang Science and Technology Project \{ZKXM[2020]4-1\}.

\section{Footnote}

Conflicts of Interest: All authors have completed the ICMJE uniform disclosure form (available at http://dx.doi. org/10.21037/qims-21-109). The authors have no conflicts of interest to declare.

Ethical Statement: The authors are accountable for all aspects of the work in ensuring that questions related to the accuracy or integrity of any part of the work are appropriately investigated and resolved. The study was conducted in accordance with the Declaration of Helsinki (as revised in 2013). Ethical approval was granted from the hospital's ethics committee, and written informed consent was provided by the patient for publication of this case report and any accompanying images. A copy of the written consent is available for review by the Editor-in-Chief of this journal.

Open Access Statement: This is an Open Access article 
distributed in accordance with the Creative Commons Attribution-NonCommercial-NoDerivs 4.0 International License (CC BY-NC-ND 4.0), which permits the noncommercial replication and distribution of the article with the strict proviso that no changes or edits are made and the original work is properly cited (including links to both the formal publication through the relevant DOI and the license). See: https://creativecommons.org/licenses/by-nc-nd/4.0/.

\section{References}

1. Kanamori N, Matsumoto Y, Oribe Y, Omi W. Multiple anomalous systemic-pulmonary connections and coronarypulmonary artery fistula associated with large aneurysms in an elderly woman. J Card Surg 2012;27:565-7.

2. Kurisu K, Hisahara M, Ando Y, Tominaga R. An unusual manifestation of brain tumor: development of delayed hemiplegia after cardiopulmonary bypass. J Card Surg 2007;22:417-8.

3. Sato M, Sai S, Matsuo S, Konishi A. Acute aortopulmonary fistula following balloon pulmonary angioplasty after arterial switch operation. Eur J Cardiothorac Surg 2016;50:188.

4. Ansari-Gilani K, Gilkeson RC, Hsiao EM, Rajiah P. Unusual Pulmonary Arterial Filling Defect caused by Systemic to Pulmonary Shunt in the Setting of Chronic Lung Disease Demonstrated by Dynamic 4D CTA. J Radiol Case Rep 2015;9:17-23.

Cite this article as: Cheng X, Zhang X, Gu F, Tian C, Wang R, Chen J, Liu J, Zeng X. Multiple systemic arteries to pulmonary artery malformations: a case description. Quant Imaging Med Surg 2021;11(11):4671-4675. doi: 10.21037/qims-21-109
5. Comaniciu D, Engel K, Georgescu B, Mansi T. Shaping the future through innovations: From medical imaging to precision medicine. Med Image Anal 2016;33:19-26.

6. Dappa E, Higashigaito K, Fornaro J, Leschka S, Wildermuth S, Alkadhi H. Cinematic rendering an alternative to volume rendering for $3 \mathrm{D}$ computed tomography imaging. Insights Imaging 2016;7:849-56.

7. Baldi D, Tramontano L, Punzo B, Orsini M, Cavaliere C. CT cinematic rendering for glomus jugulare tumor with intracranial extension. Quant Imaging Med Surg 2020;10:522-6.

8. Rowe SP, Chu LC, Fishman EK. Evaluation of Stomach Neoplasms With 3-Dimensional Computed Tomography: Focus on the Potential Role of Cinematic Rendering. J Comput Assist Tomogr 2018;42:661-6.

9. Riehl G, Chaffanjon P, Frey G, Sessa C, Brichon P-Y. Postoperative systemic artery to pulmonary vessel fistula: analysis of three cases. Ann Thorac Surg 2003;76:1873-7.

10. Voll A, Marstrander F, Wexels P. Systemic-pulmonary shunt. Dis Chest 1964;45:396-401.

11. Mager JJ, Overtoom TTC, Blauw H, Lammers JWJ, Westermann CJJ. Embolotherapy of pulmonary arteriovenous malformations: long-term results in 112 patients. J Vasc Interv Radiol JVIR 2004;15:451-6.

12. Yamanaka A, Hirai T, Fujimoto T, Hase M, Noguchi $M$, Konishi F. Anomalous systemic arterial supply to normal basal segments of the left lower lobe. Ann Thorac Surg 1999;68:332-8. 